\title{
PVInGrid: A Distributed Infrastructure for Evaluating the Integration of Photovoltaic Systems in Smart Grid
}

\author{
Lorenzo Bottaccioli $\left.^{(}\right)$, Enrico Macii, Edoardo Patti, Abouzar Estebsari, \\ Enrico Pons, and Andrea Acquaviva \\ Politecnico di Torino, Corso Duca Degli Abbruzzi 22, Turin, Italy \\ \{lorenzo.bottaccioli, enrico.macci, edoardo.patti, \\ abouzar.estebsari, enrico.pons, andrea.acquaviva\}@polito.it
}

\begin{abstract}
Planning and developing the future Smart City is becoming mandatory due to the need of moving forward to a more sustainable society. To foster this transition an accurate simulation of energy production from renewable sources, such as Photovoltaic Panels (PV), is necessary to evaluate the impact on the grid. In this paper, we present a distributed infrastructure that simulates the PV production and evaluates the integration of such systems in the grid considering data provided by smart-meters. The proposed solution is able to model the behaviour of PV systems solution exploiting GIS representation of rooftops and real meteorological data. Finally, such information is used to feed a real-time distribution network simulator.
\end{abstract}

Keywords: Photovoltaic • GIS • Distributed infrastructure • Smart-grid $\cdot$ Real-time simulation

\section{Introduction and Motivation}

Thanks to the commitment of the participating countries to Kyoto Protocol and to Paris Agreement, the presence of distributed Renewable Energy Sources (RES) is increasing in our electricity systems. In this transition from a centralized to a distributed system, is important converting passive buildings rooftops of our cities into active Photovoltaic rooftops becomes crucial. However, the deployment of PV system cannot be left to chance but needs to be planed with specific tools able to $i$ ) estimate $\mathrm{PV}$ production in time and ii) to assess the capabilities and requirements of the networks [11]. This research aims at creating PVinGRID, a distributed infrastructure composed by software and hardware components. PVinGRID integrates: i) Geographic Information Systems (GIS) data and algorithms; ii) sensor, such as weather station and smart meter; iii) realtime hardware electricity grid simulator. Exploiting GIS tools and weather station data PVinGRID estimates the availability of deployable area for PV system on rooftops and simulates sub-hourly PV generation loads in real-sky conditions. BY

(C) IFIP International Federation for Information Processing 2017

Published by Springer International Publishing AG 2017. All Rights Reserved

L.M. Camarinha-Matos et al. (Eds.): DoCEIS 2017, IFIP AICT 499, pp. 316-324, 2017.

DOI: $10.1007 / 978-3-319-56077-9 \_31$ 
merging data coming from smart meters [1] with simulated PV generation loads PVinGRID evaluates the integration of PV system in the distribution network exploiting a real-time grid simulator. The rest of the paper is organized as follows. In Sect. 2 the relationship of this research with smart system is highlighted. Section 3 presents literature methodologies and services for PV energy simulation and integration into the grid. Section 4 presents the specifications and methodology to develop our solution. In Sect. 5 the results obtained with PVinGRID are presented. Finally, Sect. 6 provides the concluding remarks.

\section{Relationship to Smart Systems}

Information and Communication Technologies (ICTs) are a key factor in the development of smart systems for Smart Energy management. They can provide useful tools for planning and monitoring the deployment of RES systems. Furthermore, the spread diffusion of heterogeneous and pervasive sensors in our houses, district and cities is increasing the smartness of hour distribution grid. With such sensors, it is possible to monitor the electricity behaviours of the users. INformation on electricity consumption are needed in both planning and monitoring phases for evaluating: i) energy management actions; ii) management of electricity distribution networks; iii) integration of renewable sources in the city. PVinGRID is a tool useful for both planning and monitoring phases of PV systems, spanning all scales starting from single building up to block, district and city. Our solution can be used for different purposes depending on the needs of different stakeholders. For instance a Single citizen uses PVinGRID for estimating the economic benefits that he/she can achieve by installing a new $\mathrm{PV}$ system. End-user wants to be aware of the avoided $\mathrm{CO}_{2}$ emission and virtuous actions to perform. Energy aggregators and Energy Communities can use our solution for scheduling the consumption pattern of their clients for maximizing self-consumption and minimizing energy bills. In particular Energy Communities can exploit such infrastructure to perform feasibility studies as proposed by [3]. Distribution system operators (DSO) can use PVinGRID for analysing the reliability of their grids after the deployment of PV system and plan, if needed, retrofits actions and/or extensions of existing distribution grids. They can exploit our solution to avoid network congestions exploiting load balancing. Lastly Energy and City planners can use PVinGRID for evaluating the benefits of large PV systems installations in the cities or monitoring the performance of already deployed PV systems. Furthermore, if accurate weather forecasts are available PVinGRID solution provides information on generation profiles of existing PV systems to: i) $\boldsymbol{D} \boldsymbol{S} \boldsymbol{O}$ for estimate possible unbalances on the grid and to evaluate load balancing actions (e.g. Demand Response and Energy Storage); ii) Market Operators for creating local or dynamic prices; iii) Smart Energy management systems for evaluating next actions in smart energy management (e.g. usage of storage and load scheduling). 


\section{State of the Art}

Geographic Information Systems (GIS) tools have been widely applied in the estimation of renewable potential as reported in [5] in particular in urban contexts for solar applications [9]. New methodologies exploiting GIS for simulation with spatial and temporal domains of PV system energy production are recently emerging $[4,10,12]$. A spatio-temporal simulation of PV production integrated with network topology and users consumption load profiles allows to estimate the real PV potential by evaluating the effects of the penetration of PV systems into the grid [4]. Available web solution such as $[13,18]$ are limited in the analysis of the time domain and do not integrate electricity consumption and network topology data. Hence, there is the need to develop frameworks that integrate consumption, production and network topology in order to evaluate the integration of PV system, or more in general RES.

Real-time simulator, such as Opal-RTß, are applied in the prototyping phase of the development of system or manufacture. This because real-time simulations allow testing solutions that are still not physical prototypes $[2,6,19]$. With respect to RES, real-time simulations have been applied for PV generation [15] and for wind-farms $[14,20]$.

In this work we propose a simulation framework called PVinGRID that couples PV simulator with a real-time grid simulator, such as Opal-RT囚. PVinGRID considers the electric behaviour of the distribution system by taking in to account both information on electricity consumption and network topology.

\section{Contributions and Innovative Aspects}

In this Section, we describe PVinGRID our distributed infrastructure for evaluating the integration of PV systems in a Smart Grid environment. PVinGRID is composed by both hardware and software components. The hardware components are: $i$ ) the real-time grid simulator, $i i)$ weather station and iii) smart meters. The software component is a distributed infrastructure for estimating PV systems energy production (see Fig. 1(a)).

\subsection{Real-Time Grid Simulator}

Real time simulation (RTS) is a highly reliable method based on electromagnetic transient simulation which serves a platform to test new control strategies or technologies on a virtual environment emulating the real world system. It provides very reliable real-like information on impacts and benefits of new strategies or devices. RTS could support decision makings from real-time operation and control phase to long-term planning. Regarding electricity systems, RTS is being widely used in protection and control system development and testing. In particular for distributed generation modelling of RES integration (e.g. PV generation penetration), and intelligent grids development. 


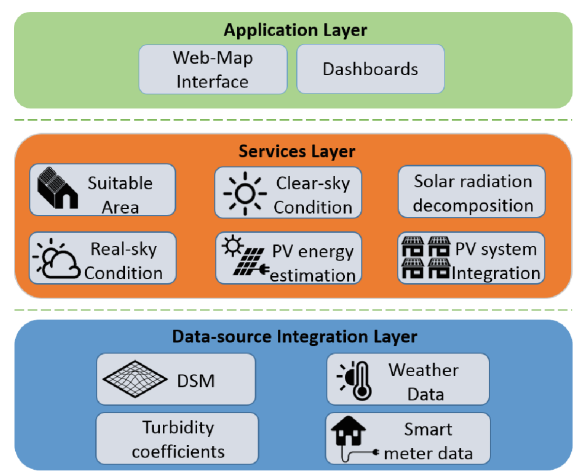

(a) Scheme of the software architecture for PV systems integration into Smart Grids

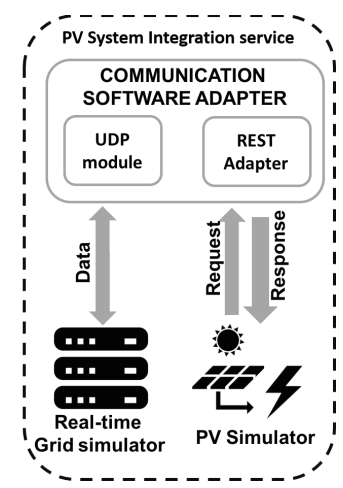

(b) Integration of PV and Real-Time grid simulators

Fig. 1. PVinGRID infrastructure

The purpose of using RTS in our work is to model a realistic distribution network to support investigations in terms of PV power penetration impacts in realworld situations. The objective is to simulate the behaviour of prosumers, and the set-up of a Software In-the-Loop (SIL) platform for laboratory validations of new control, operation, and planning algorithms for smart grids management [7].

\subsection{Photovoltaic System Simulator}

In this section we present the distributed software infrastructure for simulating PV generation profiles (see Fig. 1(a)). The development of this solution is needed to model and estimate PV system behaviours in both spatial and temporal domain. Thus, evaluations of their impact in the grid can be assessed. Our solution has been designed following both microservice ${ }^{1}$ and REST [8] approaches. This due to the intention of developing a simulation tool with an easy maintenance and to allow integration of third-party software. Our solution is composed by three layers described in the following:

The Data-source Integration Layer, the lower layer, is in charge of integrating the following data-sources: i) Digital Surface Model (DSM) of the area in study, which is a raster image that represents terrain elevation considering the presence of manufactures; ii) Linke Turbidity coefficients that express the attenuation of solar radiation related to air pollution; iii) Weather Data coming from the nearest weather station provided by third-party services; $i v$ ) electricity consumption data provided by external software platforms (eg. [16,17]).

The Services Layer is the core of the simulation infrastructure and is composed by independent services that communicates trough REST-API: Suitable

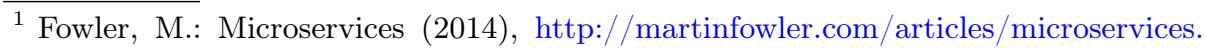
html.
} 
Area service is in charge of identifying suitable real rooftop surfaces for deploying PV system. Clear-Sky condition service exploiting the r.sun tool of GRASS$G I S^{2}$, produces a set of maps with the incident solar radiation every $15 \mathrm{~min}$ in the area of interest. Solar radiation decomposition service is used if the nearest weather station provides only global horizontal radiation (GHI). In its core, it has a set of solar decomposition models present in the literature used to estimate direct normal radiation (DNI) and diffuse horizontal radiation (DHI). Real-sky condition service produces real-sky maps for each time interval of the simulation. $P V$ energy estimation service using the maps of real-sky condition and suitable area service estimates the size of the deployable PV system and its production. It considers the cell temperature that affects the PV efficiency. PV system Integration service is in charge of correlating the simulation of PV energy production with data coming from the Smart Grid (e.g. energy consumption). This service provides information on the level of self-sufficiency (i.e. the share local demand fulfilled by PV energy production) and the level of self-consumption of the produced energy (i.e. the share of produced energy that is instantaneously consumed) for the area of interest. Furthermore, it enables the communication with the real-time grid simulator (see Sect.4.1) as described in the following Sect. 4.3.

The Application Layer provides applications that allows end-users to interact with the simulation results exploiting Web-Map interface and Dashboards.

\subsection{Smart-Grid Simulation Tool}

As mentioned in Sect. 4.2, the PV system Integration service in Fig. 1(a) correlates the output of the PV system energy simulation with information coming from the Smart Grid with the same geographic area. In addition, it enables the communication with the real-time grid simulator through the Communication Software Adapter (see Fig. 1(b)). The adapter integrates two sub-modules: i) REST adapter and ii) UDP module. The REST adapter parses the simulation request from the RTS to the PV system simulator by translating them into REST calls to remote web-services. The UDP module pushes the simulated PV energy production data in to the RTS. Furthermore, it receives and processes the information coming from the RTS.

\section{Results and Critical View}

To demonstrate an application of the proposed integrated simulation platform in PV penetration assessment, we present some results of simulating the distribution grid behaviour in a real case-study considering a high PV penetration. The area under analysis is a city district with nearly 2200 residential buildings and $43 \mathrm{MV} / \mathrm{LV}$ substations. For this purpose a summer sunny day where there is the maximum production from PV system has been selected to show the maximum

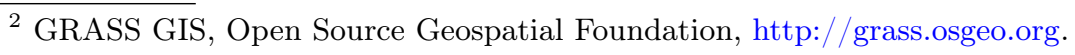




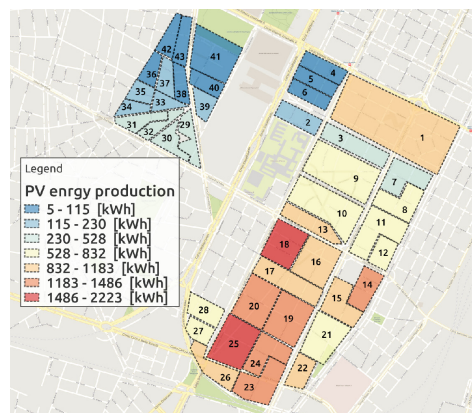

(a) Total PV energy generation in the summer sunny day

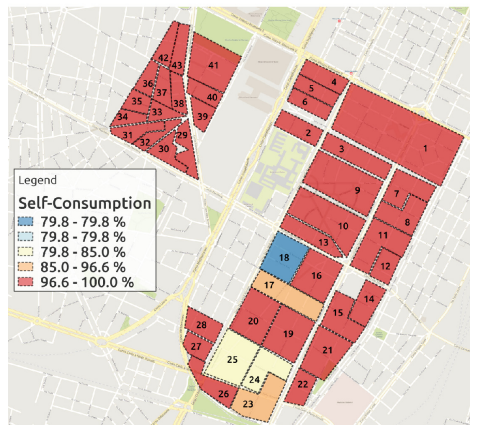

(c) Maximum Percentage of selfconsumtion of each sub-station

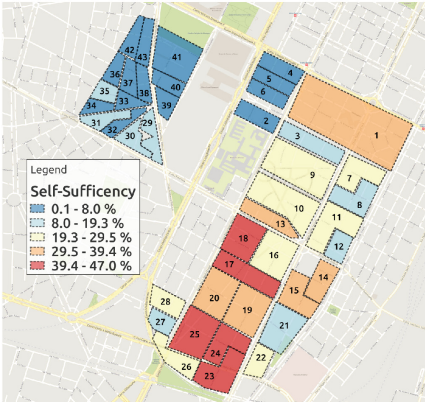

(b) Maximum Percentage of self-sufficency of each substation

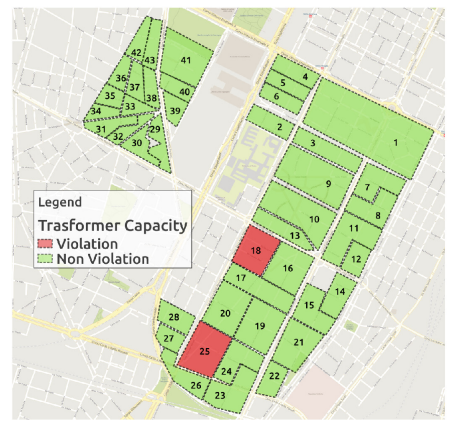

(d) Sub-station

violating

Fig. 2. Mapped simulation results

level of both self-sufficiency and self-consumption and highlighting possible violation of $\mathrm{MV} / \mathrm{LV}$ transformers. The simulation process identified 944 suitable areas in the building rooftops for deploying PV systems with a maximum nominal power equal to $14.21 \mathrm{MW}$. Figure 2(a) shows the distribution of energy production for each substation in a summer sunny day, that for the whole area is equal to $28.41 M W h$. In Fig. 2(b) and (c) the levels of self-sufficiency and selfconsumption are reported. Figure $2(\mathrm{~b})$ shows that the sub-station with less production has less than $10 \%$ of self sufficiency. However, sub-stations 17, 18, 23, 24 and 25 have a level of self-sufficiency over 39\%. Figure 2(c) revels that almost every sub-station absorbs all the produced energy from PV system. Only substations 17, 18,23, 24 and 25 feed the MV distribution grid and are the one with the highest level of self-sufficiency. Looking at the transformer capacity map (Fig. 2(d)) integrating PV generation would cause violations in sub-station 17 and 25. The maximum net consumption in these two sub-stations exceeds the transformers capacity due to an high amount of PV generation and low consumption. This highlights that in cases where PV generation is higher than local 


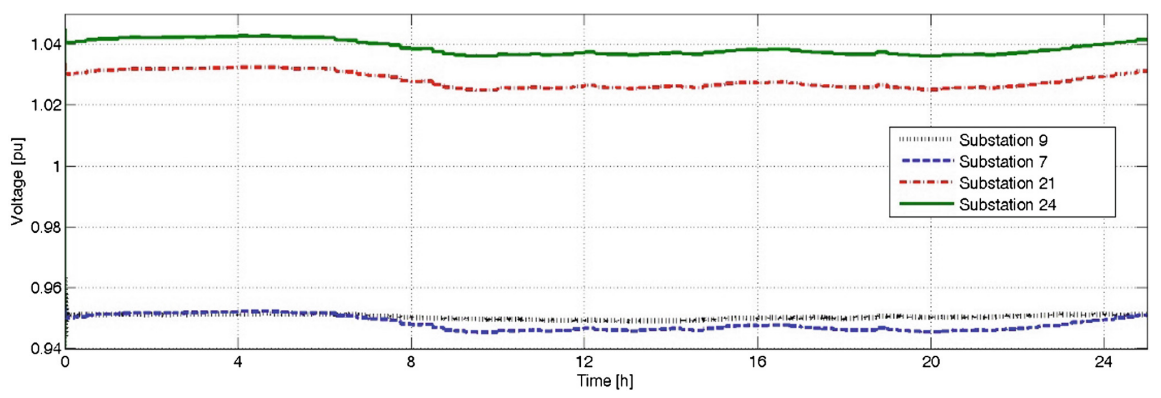

Fig. 3. Voltage profile for substations 7-9-22-24

demand the installation of PV arrays should be reduced or grid infrastructures, in terms of transformers (and also cables/lines), should be enhanced to tolerate reverse power injection from substations to the grid.

One of the other challenges due to high penetration of $\mathrm{PV}$ generation relates to the voltage control: in our case study, we observed that in two feeders which are derived from the same transformer, local generations with respect to local consumptions are not equally/closely distributed. Substations 22 and 24 with high self-sufficiency are connected to one feeder, and substations 7 and 9 with lower self-sufficiency are connected to the other feeder. According to the voltage profiles of these sub-stations (Fig. 3), any changes in the level of transformer voltage to correct over/under voltage in one feeder would result in more deviation in the other feeder.

\section{Conclusions and Future Work}

In this work PVinGRID a simulation infrastructure for evaluating PV integration in to smart grid has been presented. In addition motivations and relationships of the research with smart system has been highlighted. The results show how PVinGRID can be used for planning the deployment of PV systems and to evaluate the impact of such deployment in distribution grids. As future work a service for economic evaluation will be developed and included in the proposed solution. In particular, the simulation infrastructure will be integrated with a market simulator for evaluating dynamic prices and Demand Side Management policies. Furthermore the overall infrastructure will be used for evaluating Smart Energy actions as storage management, Demand Response events, Automatic Voltage Regulation, Network Reconfiguration and Load Balancing.

Acknowledgement. This work was supported by the EU project FLEXMETER, and by the Italian project "Edifici a Zero Consumo Energetico in Distretti Urbani Intelligenti". 


\section{References}

1. Bahmanyar, A., Jamali, S., Estebsari, A., Pons, E., Bompard, E., Patti, E., Acquaviva, A.: Emerging smart meters in electrical distribution systems: opportunities and challenges. In: Proceedings of ICEE, pp. 1082-1087 (2016)

2. Bompard, E., Monti, A., Tenconi, A., Estebsari, A., Huang, T., Pons, E., Stevic, M., Vaschetto, S., Vogel, S.: A multi-site real-time co-simulation platform for the testing of control strategies of distributed storage and V2G in distribution networks. In: Proceedings of EPE 2016 ECCE. IEEE (2016). Submitted for publication

3. Bottaccioli, L., Patti, E., Acquaviva, A., Macii, E., Jarre, M., Noussan, M.: A toolchain to foster a new business model for photovoltaic systems integration exploiting an energy community approach. In: Proceedings of ETFA2015. IEEE (2015)

4. Camargo, L.R., Zink, R., Dorner, W., Stoeglehner, G.: Spatio-temporal modeling of roof-top photovoltaic panels for improved technical potential assessment and electricity peak load offsetting at the municipal scale. Comput. Environ. Urban Syst. 52, 58-69 (2015)

5. Domínguez, J., Amador, J.: Geographical information systems applied in the field of renewable energy sources. Comput. Ind. Eng. 52(3), 322-326 (2007)

6. Dufour, C., Blanchette, H., Bélanger, J.: Very-high speed control of an FPGAbased finite-element-analysis permanent magnet synchronous virtual motor drive system. In: Proceedings of IECON 2008, pp. 2411-2416. IEEE (2008)

7. Estebsari, A., Pons, E., Patti, E., Mengistu, M., Bompard, E., Bahmanyar, A., Jamali, S.: An IOT realization in an interdepartmental real time simulation lab for distribution system control and management studies. In: Proceedings of EEEIC 2016. IEEE (2016). Submitted for publication

8. Fielding, R.T.: Architectural styles and the design of network-based software architectures. Open Source Geospatial Foundation (2000)

9. Freitas, S., Catita, C., Redweik, P., Brito, M.: Modelling solar potential in the urban environment: state-of-the-art review. Renew. Sustain. Energy Rev. 41, 915$931(2015)$

10. Jakubiec, J.A., Reinhart, C.F.: A method for predicting city-wide electricity gains from photovoltaic panels based on LiDAR and GIS data combined with hourly Daysim simulations. Sol. Energy 93, 127-143 (2013)

11. Keane, A., Ochoa, L.F., Borges, C.L., Ault, G.W., Alarcon-Rodriguez, A.D., Currie, R.A., Pilo, F., Dent, C., Harrison, G.P.: State-of-the-art techniques and challenges ahead for distributed generation planning and optimization. IEEE Trans. Power Syst. 28(2), 1493-1502 (2013)

12. Lukac, N., Seme, S., Zlaus, D., Stumberger, G., Zalik, B.: Buildings roofs photovoltaic potential assessment based on lidar (light detection and ranging) data. Energy 66, 598-609 (2014)

13. Mapdwell Solar System. http://www.mapdwell.com

14. Pak, L.F., Dinavahi, V.: Real-time simulation of a wind energy system based on the doubly-fed induction generator. IEEE Trans. Power Syst. 24(3), 1301-1309 (2009)

15. Park, M., Yu, I.K.: A novel real-time simulation technique of photovoltaic generation systems using RTDS. IEEE Trans. Energy Convers. 19(1), 164-169 (2004)

16. Patti, E., Syrri, A.L.A., Jahn, M., Mancarella, P., Acquaviva, A., Macii, E.: Distributed software infrastructure for general purpose services in smart grid. IEEE Trans. Smart Grid 7(2), 1156-1163 (2016) 
17. Patti, E., Pons, E., Martellacci, D., Castagnetti, F.B., Acquaviva, A., Macii, E.: multiflex: flexible multi-utility, multi-service smart metering architecture for energy vectors with active prosumers. In: Proceedings of SMARTGREENS, pp. 288-293 (2015)

18. Suri, M., Huld, T., Dunlop, E., Cebecauer, T.: Geographic aspects of photovoltaics in Europe: contribution of the PVGIS website. IEEE J. J-STARS 1(1), 34-41 (2008)

19. Vamsidhar, S., Fernandes, B.: Hardware-in-the-loop simulation based design and experimental evaluation of DTC strategies. In: Proceedings of PESC 2004, vol. 5, pp. 3615-3621. IEEE (2004)

20. Wang, G., Gao, W.: Real time simulation for wind power generation system using RTDS. In: Proceedings of NAPS 2008, pp. 1-6. IEEE (2008) 